08

\title{
Получение новых материалов путем сдавливания наночастиц и возбуждения химически связанных с поверхностью атомов
}

\author{
(C) А.Н. Орлов \\ Институт общей физики им. А.М. Прохорова РАН, \\ 119991 Москва, Россия \\ Центр естественно-научных исследований при институте общей фризики им. А.М. Прохорова РАН, \\ 119991 Москва, Россия \\ e-mail: orlov1@kapella.gpi.ru
}

Поступило в Редакцию 11 февраля 2019 г.

В окончательной редакции 18 сентября 2019 г.

Принято к публикации 18 сентября 2019 г.

\begin{abstract}
Показана возможность получения новых материалов за счет протекания туннельных химических реакций на поверхностях сжимаемых наночастиц. Химическая связь поверхностных атомов может быть возбуждена. Для модели были использованы кремниевые наночастицы, покрытые атомами водорода. Туннелирование атомов водорода разных наночастиц навстречу друг другу происходит под ветвями двух „пересекающихся““ парабол. Был выбран полуклассический подход. Ветви парабол направлены вверх. При сдавлении наночастиц в местах их соприкосновения давление может увеличиваться в сотни и тысячи раз. Свободные связи, появившиеся после отрыва водорода, образуют химические связи между наночастицами.
\end{abstract}

Ключевые слова: наночастицы, химические реакции под давлением, туннельные реакции.

DOI: $10.21883 / J T F .2020 .03 .48932 .49-19$

\section{Введение}

Внешнее давление и возбуждение атомов, покрывающих наночастицы, лазером или иным способом, могут существенно изменить прохождение туннельной реакции и образование новых химических связей между наночастицами. При значительном давлении и достаточном возбуждении приповерхностных атомов наночастиц возможна как безактивационная реакция объединения наночастиц, так и объединение в результате туннельной реакции. Возможно образование пористой или сплошной структуры.

В работах [1,2] было замечено, что после проведения холодного прессования порошков из наночастиц кремния в полученных пластинах (таблетках) возникают новые заметные полосы поглощения в инфракрасной области спектра. В работе [3] возникновение заметных полос поглощения в инфракрасной области спектра после проведения холодного прессования порошков объяснялось туннельными реакциями под действием давления. Предполагалось, что туннелирует атом водорода. Предполагалось также, что возможно существенное уменышение или даже исчезновение потенциальных барьеров образуются новые химические связи между атомами и молекулами, находящимися на границах в местах соприкосновения наночастиц.

В работах $[1,2]$ в качестве барьера, свозь который туннелирует только один атом, брался прямоугольный потенциальный барьер. Оценки производились для случая больших давлений. При $P S_{x 1}>E_{a}$ вероятность туннельной реакции при начальной энергии близкой к $k_{\mathrm{B}} T$ будет

$$
w=\exp \left\{-(4 / 3 \hbar)\left(2 m E_{a}\right)^{1 / 2}\left(E_{a} /\left(4 P R_{n p}^{2}\right)\right)\right\},
$$

где $P-$ внешнее давление, $R_{n p}-$ радиус наночатицы, $S$ - поперечное сечение наночастицы, $x_{1}-$ ширина прямоугольного потенциального барьера, $E_{a}$ высота барьера, $m$ - масса туннелирующего атома, $k_{\text {В }}$ - константа Больцмана, $T-$ абсолютная температура. Если в туннельной реакции участвует атом водорода, $m=1.67 \cdot 10^{-27} \mathrm{~kg}, E_{a}=6 \cdot 10^{-19} \mathrm{~J}, P=10^{8} \mathrm{~Pa}$, $R_{n p}=10 \mathrm{~nm}$, то получим $w=1.98 \cdot 10^{-5}$. Частота колебаний поверхностных хемосорбированных атомов составляет порядка $10^{13} \mathrm{~Hz}$. Туннельная реакция протекает за время $10^{-8} \mathrm{~s}$. При такой грубой оценке не ясно, возможна ли туннельная реакция для возбужденного атома поверхностного водорода и образование химической связи при условиях, приближенных к реальным.

\section{Энергетические соотношения}

При объединении двух атомов водорода, которые прежде были связаны с атомами кремния соседних наночастиц, выделяется энергия $E_{\mathrm{H}-\mathrm{H}}=4.75 \mathrm{eV}$. Предположим, что энергия связи атома водорода $E_{n-\mathrm{H}}$ с поверхностным атомом наночастицы $(n)$ равна $3 \mathrm{eV}$. Сумма этих энергий связи равна $6 \mathrm{eV}$. Тогда для прохождения туннельной реакции необходимо соблюсти условие

$$
2 E_{n-\mathrm{H}} \leq E_{\mathrm{H}-\mathrm{H}}+E_{\mathrm{H} 1}+E_{\mathrm{H} 2}+k \cdot 4 P R_{n p}^{2} \cdot \Delta x+k_{\mathrm{B}} \cdot T,
$$

где $E_{\mathrm{H} 1}$ и $E_{\mathrm{H} 2}-$ энергии возбуждения объединяющихся атомов, $k$ - доля работы давления на длине туннеля, 
которая перешла в энергию туннелирующего атома. В [1-3] предполагалось, что вся работа давления над наночастицей переходила в энергию туннелирующего атома, что вызывает сомнения. Как в настоящей работе, так и в прежних работах не учитывается работа давления до начала туннелирования.

В идеале поверхностные атомы водорода следовало бы возбуждать лазером, но, как правило, из-за отсутствия информации о частотах колебания поверхностных атомов конгломерат наночастиц нагревают и (или) сдавливают.

Пусть давление сжимающим поршнем равняется 400 at, a $k=1 / 8$. Для наночастиц с диаметром $15 \mathrm{~nm}$ при перемещении поршня $\Delta x=1.44 \cdot 10^{-11}$ m получим $P R_{n p}^{2} \cdot \Delta x / 2=0.43 \mathrm{eV}$.

\section{Возбуждение химически связанных поверхностных атомов}

Рассмотрим возбуждение поверхностного атома водорода за счет нагрева. Если $T=700 \mathrm{~K}$, то $k_{\mathrm{B}} T=0.06 \mathrm{eV}$. Пусть для валентной моды колебаний атома водорода на поверхности наночатицы $\hbar \omega_{0-1}=0.2 \mathrm{eV}$.

Вероятность возбуждения $n$-го уровня валентной колебательной моды $P_{v}(n)$ равна

$$
P_{v}(n)=\left(1-\exp \left(-\hbar \omega_{v} / k_{\mathrm{B}} \cdot T\right)\right) \cdot \exp \left(-n \hbar \omega_{v} / k_{\mathrm{B}} \cdot T\right)
$$

где индекс $v$ - принадлежность к валентной моде колебаний.

Для $T=700 \mathrm{~K}$ вероятность возбуждения второго уровня валентной моды, согласно (3), составит $1.22 \cdot 10^{-3}$. Отметим, что частота колебаний валентной моды $v_{v}=4.86 \cdot 10^{13} \mathrm{~Hz}$. Из выражения (2) следует, что

$$
\begin{aligned}
4.75 \mathrm{eV} & +0.43 \mathrm{eV}+(2 \cdot 2 \cdot 0.2) \mathrm{eV} \\
& +0.06 \mathrm{eV}=6.4 \mathrm{eV}>6.04 \mathrm{eV}
\end{aligned}
$$

Этого достаточно для прохождения туннельной реакции.

\section{Туннельные реакции}

Ниже рассмотрим только туннельные реакции. Пусть два атома водорода находятся напротив друг друга на поверхности сдавливаемых наночастиц кремния на расстоянии $1.7 \AA$ друг от друга. Амплитуда колебаний второго уровня валентной моды составит $\left(2 \hbar / m \omega_{v}\right)^{1 / 2} \approx(2+1 / 2)^{1 / 2} \approx 0.32 \AA$. Амплитуда нулевого колебания молекулы водорода составит $(4 \hbar / m \omega \mathrm{H})^{1 / 2}=0.176 \AA$. Расстояние между атомами водорода в молекуле при нулевом колебательном возбуждении равно $0.74 \AA$.

Пусть оба атома водорода на наночастице возбуждены до второго уровня и находятся симметрично на двух наночастицах. Тогда можно посчитать только вероятность туннельной реакции с одной стороны и возвести

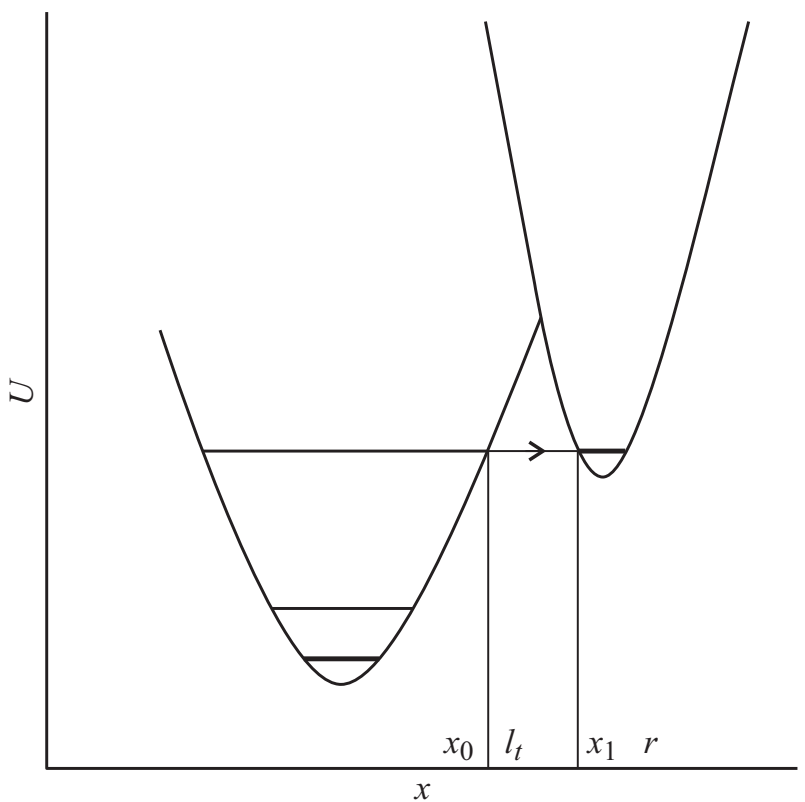

Термы атома водорода, связанного с наночастицей (левая парабола), и терм молекулы водорода (правая парабола); $x_{0}$ точка начала туннельного прохождения, $l_{t}$ - длина туннелирования, $x_{1}$ - точка окончания туннельного прохождения, $r-$ координата реакции.

эту вероятность в квадрат. Термы как атома водорода на поверхности, так и молекулы водорода приближаем параболами.

Сумма амплитуд валентной моды и расстояние между атомами водорода в молекуле водорода составит $2 \cdot 0.32 \AA+0.74 \AA+0.176 \AA=1.556 \AA$.

В таком случае длина половины туннеля, представленная на рисунке, как $l_{t}$, составит $(1.7 \AA-1.556 \AA) / 2=$ $=0.072 \AA$. Тогда $P R_{n p}^{2} l_{t}=0.43 \mathrm{eV}$. Энергия и работа, согласно формуле (2), составит $6.04 \mathrm{eV}>2 \cdot 3 \mathrm{eV}$.

Вероятность $W$ прохождения частицы, обладающей массой $m$ и энергией $E$ сквозь потенциальный барьер $U(x)$ выражается формулой

$$
W=\exp \left\{-(2 / \eta)\left\{\int_{x_{0}}^{x_{1}}(2 m(U)(x)-E)^{1 / 2} d x\right\}\right\},
$$

где $x_{0}$ и $x_{1}$ - нули функции $U(x)-E$.

Интеграл в формуле (4) берется в приближении гармонического осциллятора при наличии давления. Принимаем, что $U(x)=m \cdot \omega_{v}^{2} \cdot\left(x+x_{0}\right)^{2} / 2$, $E=m \cdot \omega_{v}^{2} \cdot x_{0}^{2} / 2+k 4 R^{2} P \cdot x$. Туннелирование атома водорода вначале идет под левой параболой, как показано на рисунке

$$
I_{1} \int_{x_{0}}^{x_{c}} \sqrt{m^{2} \omega_{v}^{2} x^{2}+2 m^{2} \omega_{v}^{2} x x_{0}-k 4 m R^{2} P x d x} .
$$

В интеграле (5) $\omega_{v}-$ круговая частота колебаний атома массы $m$ в левом параболическом потенциале, 
$k=1 / 8, x_{0}^{2}=2 \hbar(n+1 / 2) / m \omega_{v}, x_{c}-$ точка „пересечения“" парабол.

В интеграле (5) вынесем за знак интеграла $m \omega_{v}$, разделим и умножим интеграл на $x_{0}^{2}$, обозначим $x / x_{0}=y$ и тогда получим

$$
\begin{aligned}
& I_{1}=2 \hbar \cdot(n+0.5) \\
& \times \int_{1}^{y_{1}} \sqrt{y^{2}+2 y-R^{2} P /\left(8 m \omega_{v}^{3} \hbar(n+1 / 2)\right)^{1 / 2} y d y} .
\end{aligned}
$$

При больших давлениях, когда $R^{2} P /\left(8 m \omega v^{3} \hbar \times\right.$ $\times(m+1 / 2))^{1 / 2}>3$, использовать формулу (6) нельзя. Для грубой оценки можно использовать формулу (1).

Обозначим в формуле (6) $2-R^{2} P /\left(8 m \omega_{v}^{3} \hbar \times\right.$ $\times(m+1 / 2))^{1 / 2}=b_{1}, b_{1}=1.05$. Если $P=0$ или $l_{t} \cdot W_{1}$ во много раз ниже скорости движения поршня, интеграл, например $I_{1}$, и $I_{2}$ упрощается, поскольку $b_{1}=b_{2}=2$. Здесь $W_{1}$ - вероятность туннельного объединения двух поверхностных атомов водорода в газовую молекулу за одну секунду.

Выражение, стоящее под корнем в интеграле, обозначим, как A. Тогда получим выражение (7):

$$
\begin{aligned}
I_{1}= & 2 \hbar \cdot(n+0.5)\left[(1 / 4)\left(2 y+b_{1}\right) \cdot A^{0.5}\right. \\
& \left.\left.-\left(b_{1}^{2} / 8\right) \cdot \ln \right]\left(2 y+b_{1}+2 A^{0.5}\right)\right]_{1}^{y_{1}} .
\end{aligned}
$$

Для нахождения $x_{C}$ составим уравнение исходя из того, что в точке „пересечения“ термов энергии колебаний молекулы водорода и энергия колебаний валентной моды равны, но с учетом того, что нулевой колебательный уровень молекулы водорода находится точно напротив второго колебательного уровня поверхностного атома водорода, как показано на рисунке. Отсчет начнем от низшей точки левой параболы:

$$
\begin{aligned}
& m \omega_{v}^{2}\left(x_{n}+\left(5 \hbar / m \omega_{v}\right)^{1 / 2}\right)^{2} \\
= & \left(m \omega_{h}^{2} / 2\right)\left(l_{t}-x_{C}+x_{h 0}\right) 2+\hbar\left(5 \omega_{v}-\omega_{h}\right),
\end{aligned}
$$

где $\quad \omega_{h}=2 \pi \cdot 1.286 \cdot 10^{14}, \quad m=1.674 \cdot 10^{-27}$. $x_{h 0}=\left(\hbar / m \omega_{h}\right)^{1 / 2}=0.09 \AA, \quad\left((5) \cdot \hbar / m \omega_{v}\right)^{1 / 2}=0.32 \AA$, $\omega_{h} / \sqrt{2} \omega_{v}=1.87$.

Из (8) получим $x_{C}=0.043 \AA, l_{t}-x_{C}=0.029 \AA$, тогда $y_{1}=1.13, y_{2}=1.32$. Подставив в формулу (7) $y_{1}$, получим, что интеграл (7) равен $0.99 \hbar$.

На рисунке обозначены $x_{0}$ - точка начала туннельного прохождения, $l_{t}$ - длина туннелирования, $x_{1}$ - точка окончания туннельного прохождения, $r-$ координата реакции.

Возьмем интеграл под термом (параболой) молекулы водорода:

$$
I_{2}=\hbar \cdot \int_{1}^{y_{2}} \sqrt{y^{2}+2 y-R^{2} P /\left(8 m \omega_{h}^{3} \hbar(n+1 / 2)\right)^{1 / 2} d y} .
$$

Формула (9) - это интеграл под правой параболой на рисунке, $b_{2}=1.5$. Этот интеграл такой же, как в формуле (8), но п равняется нулю, и интеграл берется в других пределах.

Интеграл (9) равен 0.35ћ. Интеграл по всей длине туннеля равен 1.34ћ. Вероятность туннельной реакции с одной стороны равна $W_{1 / 2}=\exp (-2 \cdot 1.34)=6.86 \cdot 10^{-2}$. Полная вероятность образования молекулы водорода $W_{\mathrm{O}}$ будет равна $W_{\mathrm{O}}=\exp (-4 \cdot 1.34)=4.7 \cdot 10^{-3}$.

С учетом вероятности возбуждения получим вероятность $W_{1}$ за одну секунду:

$$
\left(4.76 \cdot 10^{-3} \cdot\left(1.22 \cdot 10^{-3}\right)^{2} \cdot 4.86 \cdot 10^{13}\right) / \mathrm{s}=3.44 \cdot 10^{5} / \mathrm{s} .
$$

Понятно, что вероятность $W_{1}$ с учетом других возможных возбуждений будет больше.

\section{Выводы}

1. Предложен принципиально новый подход в расчете туннельных реакций для наночастиц под давлением. Частица проходит под своей колебательной параболой и под ветвью нового терма и переходит от одного терма к другому.

2. За счет давления атомы водорода разных наночастиц могут сблизиться на несколько и даже на доли ангстрем. В результате могут происходить туннельные реакции объединения атомов в молекулы водорода.

3. Для температуры в $700 \mathrm{~K}$ применение квазиклассического приближения дает высокую вероятность прохождения туннельной реакции - объединения атомов водорода, прежде химически связанных с наночастицами, в молекулы водорода.

\section{Конфликт интересов}

Автор заявляет, что у него нет конфликта интересов.

\section{Список литературы}

[1] Kuz'min G.P., Kononov N.N., Tikhonevitch O.V., Surkov A.A., Khokhlov E.M. Optical and electrical properties of thin wafers produced from nanocrystalline Si powders. Amorphous and microcrystalline semiconductors. 2004, Intern. Conf. St-Petersburg, Russia, 5-7 July, 2004, P. 166-167.

[2] Kuz'min G.P., Kononov N.N., Orlov A.N., Tikhonevitch O.V., Surkov A.A. // Semicond. Phys. 2005. Vol. 39. N 7. P. 835-839.

[3] Kononov N.N., Kuz'min G.P., Orlov A.N., Surkov A.A., Tikhonevich O.V., Khokhlov E.M. // Laser Phys. 2006. Vol. 15. N 11. P. 217-225. 